\title{
EARLY DISCHARGE AFTER CORONARY ARTERY BYPASS GRAFT SURGERY: ARE PATIENTS REALLY GOING HOME EARLIER?
}

Harold L. Lazar, MD

Carmel A. Fitzgerald, RN, MS

Tazeen Ahmad, AB

Yusheng Bao, MD

Theodore Colton, $\mathrm{ScD}$

Oz M. Shapira, MD

Richard J. Shemin, MD
Objective: This study was undertaken to determine whether early discharge after coronary artery bypass grafting allows patients to return home earlier or merely increases the use of outpatient nursing and inpatient rehabilitation services.

Methods: Patterns of discharge were analyzed in 407 patients undergoing bypass grafting in 1990, when there were no early extubations or fast track protocols, and compared with 379 patients in 1998, when these protocols were used.

Results: Patients in 1998 had a higher prevalence of class IV angina (35.3\% vs $22.8 \% ; P=.006)$, urgent/emergency surgery $(58.3 \%$ vs $44.9 \% ; P=.015)$, and lower ejection fractions $(48.9 \% \pm 16.4 \%$ vs $52.9 \% \pm 13.5 \% ; P=.0002)$. Despite these increased risk factors, 1998 patients spent less time receiving ventilatory support $(10.2 \pm 9.2$ vs $26.7 \pm 15.7$ hours; $P<.001)$ and had a shorter length of stay ( $5.4 \pm 2.5$ vs $9.2 \pm 4.3$ days; $P<.001)$. However, fewer 1998 patients were discharged home $(56.7 \%$ vs $97.0 \% ; P<.0001)$. A higher percentage of 1998 patients $(43.3 \%$ vs $2.9 \% ; P<.00001)$ were discharged to extended care facilities where their average length of stay was $10.6 \pm 15.1$ days. Readmission to the Boston Medical Center was also more common in 1998 patients $(5.3 \%$ vs $0.5 \% ; P<.0001)$.

Conclusions: Early extubation and fast track protocols have resulted in earlier discharge from acute care facilities. However, the anticipated earlier return to home has been offset by the increased use of outpatient nursing services, discharges to extended care facilities, and hospital readmissions. ( $\mathrm{J}$ Thorac Cardiovasc Surg 2001;121:943-50)
C hanges in reimbursement policies and rising health care costs have mandated that surgeons reduce the costs of coronary artery bypass graft (CABG) surgery. One method of reducing costs is to limit the hospital length of stay (LOS). Fast track protocols, same-day admission and early extubation programs, and critical pathways have all been successful in significantly reducing hospital LOS. ${ }^{1-12}$ This has contributed to decreased hospital costs for patients undergoing CABG surgery. $1,2,10,12$

From the Department of Cardiothoracic Surgery, The Boston Medical Center and the Boston University School of Medicine, Boston, Mass.

Read at the Eightieth Annual Meeting of The American Association for Thoracic Surgery, Toronto, Ontario, Canada, April 30-May 3, 2000.

Copyright (C) 2001 by The American Association for Thoracic Surgery

0022-5223/2001 $\$ 35.00+0 \quad \mathbf{1 2 / 6 / 1 1 3 7 5 1}$

doi:10.1067/mtc. 2001.113751
Although fast track protocols and critical pathways have contributed to decreased LOS in acute care hospitals, it is unclear whether patients are being discharged home earlier. If early discharge from acute care facilities results in increased admissions to extended care facilities and increased readmission rates, then earlier discharge to the home environment may not be achieved. This study was therefore undertaken to determine whether early discharge after CABG surgery from acute care facilities results in earlier return to the home environment or merely the use of outpatient nursing and inpatient rehabilitation services.

\section{Methods}

The patterns of discharge after isolated CABG surgery at the Boston Medical Center were analyzed in 407 patients from January 1, 1990, to December 31, 1990, when there was no targeted LOS, no fast track and early extubation protocols or critical pathways, and same-day admission programs. This group was compared with patients undergoing isolated CABG 
Table I. Patient profiles

\begin{tabular}{lccc}
\hline Variable & 1990 & 1998 & P value \\
\hline $\mathrm{N}$ & 407 & 379 & .79 \\
Age (y) & $64.8 \pm 9.8$ & $64.8 \pm 11.6$ & .25 \\
Female sex (\%) & $116(28.5)$ & $94(24.8)$ & .006 \\
Class IV/unstable angina (\%) & $89(22.8)$ & $134(35.3)$ & .80 \\
CHF $(\%)$ & $101(24.8)$ & $92(24.2)$ & .001 \\
MI $(\%)$ & $265(65.1)$ & $193(50.9)$ & .32 \\
Hypertension (\%) & $260(63.8)$ & $255(67.2)$ & .09 \\
AODM (\%) & $117(38.7)$ & $134(35.3)$ & .04 \\
CVA (\%) & $23(5.6)$ & $10(2.6)$ & .03 \\
Vessels $\geq 70 \%$ stenosis & $3.1 \pm 1.0$ & $3.3 \pm 0.9$ & .0002 \\
EF $(\%)$ & $52.9 \pm 13.5$ & $48.9 \pm 16.4$ & .015 \\
Urgent/emergency (\%) & $183(44.9)$ & $221(58.3)$ & .0001 \\
LOS before CABG (d) & $3.2 \pm 3.7$ & $1.9 \pm 3.8$ & \\
\hline
\end{tabular}

Values are mean \pm standard deviation. $C H F$, Congestive heart failure; $M I$, myocardial infarction; AODM, adult-onset diabetes mellitus; $C V A$, cerebrovascular accident; $E F$, ejection fraction; $L O S$, length of stay; $C A B G$, coronary artery bypass graft.

from January 1, 1998, to December 31, 1998, when these protocols were in place, in an attempt to discharge patients by the fifth postoperative day. Postoperative in-hospital mortalities (12 [2.9\%] 1990 vs 0 [0\%] 1998) and patients hospitalized for 30 days or more after CABG (5 [1.2\%] 1990 vs 0 [0\%] 1998) were excluded from the study.

All surgical procedures were performed with a membrane oxygenator at systemic temperatures of $35^{\circ} \mathrm{C}$ using multidose cold blood cardioplegic solution, given antegradely, retrogradely, or in an antegrade/retrograde fashion at the surgeon's discretion. Similarly, distal anastomoses were performed either under 1 single crossclamp period or after construction of the proximal anastomosis in the beating heart at the surgeon's preference. All patients in 1998 received heparinbonded circuits. A balanced anesthetic technique was used with increased use of inhalational agents and lower dosages of narcotics in 1998. Inotropic agents were used to maintain a cardiac index of $2.0 \mathrm{~L} \cdot \mathrm{min}^{-1} \cdot \mathrm{m}^{-2}$.

Patients undergoing CABG in 1998 were targeted for discharge on the fifth postoperative day. Patients were extubated within 6 to 8 hours after CABG and were transferred from the intensive care unit on the first postoperative day, provided that vasodilators, inotropic drugs, or vasoconstricting agents were not necessary. $\beta$-Blockers were instituted in all patients on the first postoperative day. Corticosteroids and triiodothyroxine were not administered postoperatively unless the patients had been receiving these medications before CABG. Fast track protocol and critical pathways were used in 1998 to target discharge after CABG by the fifth postoperative day.

Criteria for discharge from the hospital to home included a stable cardiac rhythm, an oral temperature of less than $37.5^{\circ} \mathrm{C}$ $\left(99^{\circ} \mathrm{F}\right)$, a hematocrit value of $25 \%$ or more, oral intake of at least 1000 calories per day, successful completion of an exercise test that included independent ambulation and the ability to climb one flight of stairs, no significant wound complications, and adequate home support systems. Patients not meeting these criteria were referred to extended care facilities. The individual surgeon was responsible for determining whether a patient should be sent home, have the hospital stay extended, or be referred to an extended care facility. The particular extended care facility chosen (rehabilitation center, transitional care unit, skilled nursing facility) was determined by the medical condition and rehabilitation needs of the patient, along with the patient's insurance coverage. All patients were seen by the surgeon 2 weeks after discharge to the home or 2 weeks after discharge from an extended care facility.

Clinical variables expected to contribute to morbidity and mortality were selected for analysis and defined by means of the Conditions of Terms of the Society of Thoracic Surgeons (STS) National Cardiac Surgery Database. ${ }^{13}$

All values represent the mean \pm standard deviation. Differences in categorical data were compared by means of the $\chi^{2}$ or Fisher exact test where appropriate. Data with numerical responses were analyzed with the Student $t$ test. Analysis of variance was used to determine differences in operative risk, LOS, and discharge disposition among individual surgeons. Multivariate analyses were conducted with conditional logistic regression techniques to assess the predictors for discharge to extended care facilities. All tests were 2-sided.

\section{Results}

Preoperative risk factors. Patient profiles between patients undergoing CABG in 1990 and 1998 appear in Table I. Patients who received their CABG in 1998 had a higher prevalence of class IV and unstable angina (22.8\% vs $35.3 \% ; P=.006)$ and were more likely to require urgent or emergency surgery (44.9\% vs $58.3 \%$; $P=.015)$. They had a decreased prevalence of recent or remote myocardial infarctions $(65.1 \%$ vs $50.9 \%$; $P=.001)$ and strokes (5.6\% vs $2.6 \% ; P=.04)$. Patients undergoing $\mathrm{CABG}$ in 1998 also tended to have lower ejection fractions $(52.9 \% \pm 13.5 \%$ vs $48.9 \% \pm 16.4 \%$; $P=.0002)$ and had more critical coronary artery lesions (vessels $\geq 70 \%$ stenoses; $3.1 \pm 1.0$ vs $3.3 \pm 0.9 ; P=.03$ ). 
Table II. Postoperative results

\begin{tabular}{lccc}
\hline Variable & 1990 & 1998 & P value \\
\hline $\mathrm{N}$ & 407 & 379 & $<.001$ \\
Time to extubation (h) & $26.7 \pm 15.7$ & $10.2 \pm 9.2$ & .84 \\
Inotropic support (\%) & $34(8.3)$ & $34(8.9)$ & .33 \\
IABP support (\%) & $18(4.4)$ & $23(6.0)$ & .065 \\
Reoperation for bleeding (\%) & $9(2.2)$ & $2(0.5)$ & .24 \\
MI (\%) & $9(2.2)$ & $4(1.0)$ & .44 \\
Sternal infections (\%) & $9(2.2)$ & $6(1.5)$ & .94 \\
CVA (\%) & $4(0.9)$ & $5(1.3)$ & .83 \\
Pneumonia/respiratory insufficiency (\%) & $20(5.2)$ & .43 \\
Renal dysfunction (\%) & $23(5.7)$ & $8(2.1)$ & .19 \\
Atrial fibrillation (\%) & $4(0.9)$ & $113(29.8)$ & $<.001$ \\
LOS (d) & $139(34.1)$ & $5.4 \pm 2.5$ & \\
\hline
\end{tabular}

Values are mean \pm standard deviation. IABP, Intra-aortic balloon pump; $M I$, myocardial infarction; CVA, cerebral vascular accident; $L O S$, length of stay.

Operative results. Patients undergoing CABG in 1998 had more vessels bypassed ( $3.2 \pm 0.9$ vs $3.4 \pm 0.9$; $P=.039)$ and longer crossclamp times $(36.7 \pm 13.2$ minutes vs $49.3 \pm 20.1$ minutes; $P<.0001)$. The longer crossclamp times were a reflection of the technique by which both proximal and distal anastomoses are performed during a single crossclamp period. There was, however, no difference in cardiopulmonary bypass time $(85.7 \pm 28.9$ minutes vs $87.6 \pm 49.9$ minutes; $P=.52)$.

Postoperative results. Postoperative results are given in Table II. As a result of early extubation protocols, patients in 1998 were extubated in less time as those in $1990(26.7 \pm 15.7$ hours vs $10.2 \pm 9.2$ hours; $P<.001)$. Both groups had similar frequencies of inotropic and intra-aortic balloon pump support, reoperation for bleeding, myocardial infarctions, strokes, sternal wound infections, respiratory complications, and atrial fibrillation.

LOS. The institution of fast track and early extubation programs, targeted LOS, and critical pathways helped to reduce LOS substantially. Patients in 1998 spent an average of 3.8 fewer days in the hospital after the operation than in $1990(9.2 \pm 4.3$ days vs $5.4 \pm 2.5$ days; $P<.001$ ). As a result of same-day admission programs, which allowed the discharge of patients having elective CABG after their catheterization, patients spent less time in the hospital before CABG surgery. The result was a decrease in pre-CABG LOS between 1990 and $1998(3.2 \pm 3.7$ days vs $1.9 \pm 3.8$ days; $P=$ .0001).

Stepwise multiple linear regression analyses were performed to determine what independent variables were most important in determining LOS. Significant predictors of LOS included age $(P<.001$; parameter estimate $=0.066)$, urgent/emergency surgery $(P<.01$; parameter estimate $=-0.638$ ), adult-onset diabetes mellitus $(P<.02$; parameter estimate $=0.564)$, and a history of a myocardial infarct (recent or remote; $P<.02$; parameter estimate $=0.73$ ). The most important predictor of LOS in these data was the year that the operation was performed $(P<.0001$; parameter estimate $=3.800$ ).

Discharge disposition. The decreased LOS was achieved in part by the increased use of outpatient nursing and inpatient extended care services (Table III). In $1990,97 \%$ of patients were discharged home as opposed to only $56 \%$ in $1998(P<.001)$. Furthermore, only $15 \%$ of patients were sent home with services in 1990, whereas nearly $47 \%$ of 1998 patients had home services $(P<.001)$. In 1990 , only $3 \%$ of patients were discharged to an extended care facility as opposed to $43 \%$ in $1998(P<.001)$.

In $1990,100 \%$ of patients requiring post-hospitalization care went to rehabilitation facilities, which provided both acute and chronic care. In 1998, only 3\% of patients went to rehabilitation facilities; the vast majority, $47.5 \%$, went to transitional care units with $20.7 \%$ going to skilled nursing facilities. Patients who went to extended care facilities spent a considerable amount of time there, $13.0 \pm 11.6$ days in 1990 and $10.6 \pm 15.1$ days in 1998.

Using multiple logistic regression, we were able to identify 8 factors predictive of discharge to an extended care facility (Table IV). Patients discharged to an extended care facility had more vessels bypassed, were nearly 10 years older, tended to be female, and had a recent or remote myocardial infarction. They were almost twice as likely to have diabetes, had lower ejection fractions, and tended to have longer LOS after their CABG. Furthermore, individual surgeons were also responsible for determining which patients were more likely to go to extended care facilities. 
Table III. Discharge disposition

\begin{tabular}{lccc}
\hline Variable & 1990 & 1998 & P value \\
\hline $\mathrm{N}$ & 407 & 379 & \\
Home (\%) & $335(82.3)$ & $38(10.0)$ & $<.001$ \\
Home with services (\%) & $60(14.7)$ & $177(46.7)$ & $<.001$ \\
Rehabilitation unit (\%) & $12(2.9)$ & $52(13.7)$ & $<.001$ \\
TCU (\%) & 0 & $78(20.5)$ & $<.001$ \\
SNF (\%) & 0 & $34(8.9)$ & $<.001$ \\
Readmit to BMC (\%) & $2(0.5)$ & $20(5.3)$ & $<.001$ \\
LOS extended care (d) & $13.0 \pm 11.6$ & $10.6 \pm 15.1$ & .23 \\
\hline
\end{tabular}

Values are mean \pm standard deviation. TCU, Transitional care unit; SNF, skilled nursing facility; BMC, Boston Medical Center; $L O S$, length of stay.

Table IV. Factors responsible for discharge to extended care facilities in 1998

\begin{tabular}{lccccc}
\hline Variable & Home & Extended care & OR & $95 \%$ CL & P value \\
\hline No. vessels bypassed & $3.2 \pm 0.9$ & $3.4 \pm 0.9$ & 0.848 & $0.637-1.127$ & .039 \\
Age (y) & $60.5 \pm 10.3$ & $70.5 \pm 10.6$ & 1.116 & $1.082-1.151$ & $<.001$ \\
Female sex (\%) & $31(14.4)$ & $63(38.6)$ & 2.768 & $1.488-5.152$ & $<.001$ \\
MI (\%) & $99(46.0)$ & $98(59.9)$ & 1.990 & $1.115-3.554$ & $<.001$ \\
AODM (\%) & $56(26.0)$ & $78(47.6)$ & 2.587 & $1.470-4.554$ & $<.001$ \\
EF $(\%)$ & $51.3 \pm 15.7$ & $45.7 \pm 16.7$ & 0.981 & $0.964-0.999$ & $<.004$ \\
LOS after CABG (d) & $5.0 \pm 1.9$ & $6.0 \pm 2.9$ & 1.381 & $1.166-1.636$ & .001 \\
Surgeon A & $56(56.5)$ & $43(43.4)$ & 4.145 & $1.733-9.911$ & - \\
Surgeon C & $62(47.3)$ & $69(52.6)$ & 6.893 & $2.922-16.258$ & - \\
Surgeon D & $37(61.6)$ & $23(38.3)$ & 1.917 & $0.760-4.836$ & - \\
\hline
\end{tabular}

Values are mean \pm standard deviation. Surgeons A, C, and D have been compared with surgeon B to determine odds ratio $(O R)$ and $95 \%$ confidence limits $(C L)$. $M I$, Myocardial infarction; $A O D M$, adult-onset diabetes mellitus; $E F$, ejection fraction; $L O S$, length of stay; $C A B G$, coronary artery bypass graft.

Table V summarizes the patterns of discharge by individual surgeons in 1998. Although there was no difference in operative risk, as judged by the STS expected mortality, there was a significant difference in the post-CABG LOS and frequency of discharge to extended care facilities among the surgeons. Surgeons with the shortest LOS had the highest percentage discharge to extended care facilities, whereas the surgeon with the longest LOS had the lowest frequency of discharge to extended care facilities.

Although LOS was significantly shorter for patients undergoing CABG in 1998, the readmission rate to the Boston Medical Center was 10 times greater $(0.5 \%$ vs 5.3\%; $P<.001$; Table III).

Factors responsible for discharge to extended care facilities in 1998. The factors responsible for discharge to extended care facilities in 1998 are summarized in Table VI. The most common reason was a medical condition $(35.9 \%)$, the most frequent being respiratory issues (39/59 patients). The next most common condition was related to functional status $(27.4 \%)$, which consisted of deconditioning, weakness, and inability to ambulate. Social conditions compromised $15.2 \%$ of all patients and consisted of inadequate home support ser- vices and situations in which the patient had no one else at home. In $21.3 \%$ of patients, there was no definitive reason for transfer to an extended care facility.

\section{Discussion}

In an attempt to control the cost of CABG surgery, numerous changes have been instituted that have had a substantial impact on LOS. Patients having elective surgery are now admitted on the same day of surgery, significantly decreasing hospital stay and costs. ${ }^{1,12}$ Anesthetic techniques avoid high-dose narcotic agents and use more inhalational agents. Cell saving techniques have resulted in lower transfusion requirements and less weight gain. Both these modifications have resulted in earlier extubation and faster discharge from the intensive care unit. Heparin-bonded circuits using low-dose heparin have also minimized fluid gain and transfusion requirements, decreased the incidence of ischemic events, and have been shown to significantly decrease LOS. ${ }^{14}$ The most dramatic changes have occurred in postoperative management. Early extubation, fast tracking, and critical pathways have significantly reduced hospital LOS. The introduction of these policies at the Boston Medical Center reduced the 
Table V. Patterns of discharge by individual surgeons in 1998

\begin{tabular}{lcccc}
\hline & \multicolumn{3}{c}{ Surgeon } \\
\cline { 2 - 5 } & $A$ & $B$ & $C$ & $D$ \\
\hline STS database expected mortality & 4.16 & 4.83 & 4.29 & 3.33 \\
LOS (d) & $4.9 \pm 2.4$ & $6.8 \pm 2.4^{*}$ & $4.9 \pm 2.3$ & $5.2 \pm 2.3$ \\
Discharge to extended care facility (\%) & $42(43.4)$ & $29(32.5) \dagger$ & $69(52.6)$ & $23(38.3)$ \\
Discharge home (\%) & $56(56.5)$ & $60(67.4) \dagger$ & $62(47.3)$ & $37(61.6)$ \\
\hline
\end{tabular}

Values are mean \pm standard deviation. STS, Society of Thoracic Surgery; LOS, length of stay.

$* P<.0001$ from surgeons A, C, and D.

$\dagger P<.009$ from surgeon $\mathrm{C}$.

mean LOS from 9.2 to 5.4 days $(P<.001)$ from 1990 to 1998. Similar results have been achieved in other centers ${ }^{2-4,7,10,11}$ and in both older patients ${ }^{6,8}$ and nonacademic rural areas. ${ }^{9}$ This reduction in LOS has contributed to decreased costs for acute care facilities and has been achieved without a significant increase in morbidity or mortality. $2,7,10,11$

Our study suggests, however, that this apparent decreased LOS has been achieved by increased use of home services and extended care facilities. In 1990, only $3 \%$ of patients undergoing CABG at the Boston Medical Center were discharged to extended care facilities and only $15 \%$ received outpatient nursing services. Similar results were seen in 2 large studies involving Medicare CABG patients before fast tracking, in which the incidence of discharge to extended care facilities was only $4 \% .^{15,16}$ In contrast, in 1998, $43 \%$ of patients undergoing CABG at the Boston Medical Center were discharged to extended care facilities and $47 \%$ of patients had home nursing services. Although fast tracking protocols have resulted in earlier home discharge for good-risk patients, they have resulted in an increased use of ancillary services and extended care facilities for patients who cannot be discharged to their homes.

Our study identified several factors that may be predictive of those patients who require discharge to extended care facilities. These patients were more likely female and were nearly 10 years older than those discharged home. They had a higher frequency of recent or remote myocardial infarctions and diabetes. They also had lower ejection fractions. Not surprisingly, these are the same factors that we and others have previously found predictive of longer hospital LOS. ${ }^{5,15,17,18}$ In our study, we also found that the individual surgeon constitutes an independent factor in determining the discharge disposition of patients. In a review of the Duke database that examined factors influencing the cost of CABG surgery, Smith, Smith, and Muhlbaier ${ }^{17}$ found that the individ-
Table VI. Factors responsible for discharge to extended care facilities in 1998

\begin{tabular}{lrl}
\hline Variable & $N$ & \\
\hline Medical (\%) & & $59(35.9)$ \\
$\quad$ Respiratory & 39 & \\
$\quad$ Psychiatric & 2 & \\
$\quad$ Wound related & 8 & \\
$\quad$ Renal/dialysis & 5 & \\
$\quad$ Neurologic & 5 & $45(27.4)$ \\
Functional (\%) & & \\
$\quad$ Impaired ambulation & 13 & \\
$\quad$ Deconditioned, weak & 32 & \\
Social (\%) & & \\
$\quad$ Lives alone & 15 & \\
$\quad$ Inadequate home support services & 10 & $35(21.3)$ \\
Indeterminate (\%) & & \\
\hline
\end{tabular}

ual surgeon was an independent factor in determining CABG costs. They also found that the predicted mortality rates were equal among the surgeons, so that the costs were not reflective of patient risk. Anderson and his coworkers, ${ }^{1}$ in a study investigating the selection of patients for same-day CABG procedures, found that the individual surgeon was an independent predictor of whether or not patients were admitted on the day of CABG. These differences could not be explained by risk factors alone. We note similar findings with our own data. Although there was no difference in operative risk among the 4 surgeons, there was a significant difference in their LOS and frequency of discharge to extended care facilities. The surgeon with the shortest LOS had the highest discharge rate to extended care facilities, whereas the surgeon with the longest LOS discharged significantly fewer patients to extended care facilities. This suggests that, in an attempt to decrease LOS in acute care hospitals, individual surgeons may increase their use of extended care facilities.

Although it is entirely appropriate to use extended care facilities for patients who cannot be discharged 
home in a timely fashion, these facilities may actually consume more health care resources than those in the acute care setting. In addition to the daily inpatient costs, other changes include medical and cardiology consultations, nutritionists, physical therapists, social workers, psychiatrists, and rehabilitation specialists. Furthermore, the LOS in extended care facilities may be prolonged. The average LOS was 13.0 days in 1990 and 10.6 days in 1998. In addition, the increased use of extended care facilities did not preclude readmission to the Boston Medical Center. We found that patients treated in 1998 were nearly 10 times more likely to be readmitted after discharge than those treated in 1990. We realize that the readmission rate to our own medical center underestimates the actual incidence of readmissions to acute care hospitals after CABG. In many instances, readmissions to community hospitals are not always communicated by local physicians. Furthermore, the criteria for readmission to local hospitals do not necessarily reflect those used at our own institution.

Our study is limited in that we have only reported on patients from a single tertiary medical center. Furthermore, our practice has a high percentage of older, high-risk patients requiring urgent $\mathrm{CABG}$, for whom use of extended care facilities is more likely. Because of the wide discrepancies in charges among the various extended care facilities and negotiated costs among health maintenance organizations (HMOs), actual costs were not reported. Can we, therefore, state that care shifting is the same as cost shifting? The figures we obtained from one of our largest HMOs helps to illustrate our point. This particular HMO pays $\$ 1200.00$ per day for an acute care hospital bed, $\$ 300.00$ for a skilled nursing facility, $\$ 450.00$ for a transitional care unit, and $\$ 950.00$ for a full-service rehabilitation unit. Our average LOS was 5 days for an acute care hospital and 10 days for an extended care facility. On the basis of these figures, keeping a patient in an acute care facility for an additional 2 days and then discharging the patient home would increase the costs by $\$ 2400.00$. However, if that same patient was discharged on postoperative day 5 but spent 10 days in an extended care facility, the cost would be increased to $\$ 3000.00$ in a skilled nursing facility, $\$ 4500.00$ in a transitional care facility, and $\$ 9500.00$ in a rehabilitation unit.

Our study suggests some ways in which overall LOS after CABG may be shortened and the need for extended care facilities diminished. Nearly $15 \%$ of patients required extended care because of inadequate living arrangements. Preoperative consultation with social service personnel may help with better identification of these patients so that earlier arrangements for a more stable home environment can be instituted. Nearly $21 \%$ of patients had no definitive reason for discharge to an extended care facility. These patients usually fail to meet the requirements for discharge on the fifth postoperative day but might be more suitable for discharge on day 6 or 7. Extending the hospitalization of these patients for an additional 24 to 48 hours may allow their discharge home and avoid a more costly stay in an extended care facility. Finally, our study indicates that LOS in extended care facilities is nearly double that for acute care facilities. The fast track protocols and critical pathways that have been so successful in decreasing CABG costs in acute care facilities merit implementation in our extended care facilities.

In conclusion, we have shown that the anticipated earlier return to home thought to be derived from earlier discharge after CABG surgery is offset by increased use of extended care facilities and more frequent readmissions to tertiary care hospitals. The increased use of both inpatient and outpatient services also has the potential to increase the cost of CABG surgery. If we are to realize cost savings from early discharges, we must extend those programs and protocols so successful in reducing costs in acute care facilities to include the outpatient and inpatient extended care facilities to which our patients are discharged.

Received for publication May 4, 2000; revisions requested July 17, 2000; revisions received Nov 30, 2000; accepted for publication Dec 5, 2000.

Address for reprints: Harold L. Lazar, MD, Department of Cardiothoracic Surgery, Boston Medical Center, Suite B404, 88 E Newton St, Boston, MA 02118 (E-mail: harold.lazar@bmc.org).

\section{REFERENCES}

1. Anderson RP, Guyton SW, Paull DR, Tidwell SL. Selection of patients for same-day coronary bypass operations. J Thorac Cardiovasc Surg 1993;105:444-52.

2. Cohn LH, Rosborough D, Fernandez J. Reducing costs and length of stay and improving efficiency and quality of care in cardiac surgery. Ann Thorac Surg 1997;64:S58-60.

3. Engelman RM, Rousou JA, Flack SE, Deaton DW, Humphrey $\mathrm{CB}$, Ellison LH, et al. Fast track recovery of the coronary bypass patient. Ann Thorac Surg 1994;58:1742-6.

4. Krohn BG, Kay JH, Mendez MA, Zubiate P, Kay GL. Rapid sustained recovery after cardiac operations. J Thorac Cardiovasc Surg 1990;100:194-7.

5. Lazar HL, Fitzgerald C, Gross S, Heeran T, Aldea GS, Shemin RJ. Determinants of length of stay after coronary artery bypass graft surgery. Circulation 1995;92(Suppl):II-20-4.

6. Lee JH, Swain B, Andrey J, Murrell HK, Geha AS. Fast track recovery of elderly coronary bypass surgery patients. Ann Thorac Surg 1999;68:437-41.

7. Nikas DJ, Freeman JE, Luterman AR, Warnken LC, Nenstiel RD, Farrell M, et al. Use of a national database to assess perioperative 
risk, morbidity, mortality, and cost savings in coronary artery bypass grafting. South Med J 1996;89:1074-7.

8. Paone G, Higgins RS, Haustad SL, Silverman NA. Does age limit the effectiveness of clinical pathways after coronary artery bypass graft surgery? Circulation 1998;98(Suppl):II-41-5.

9. Quigley RL, Reitknecht FL. A coronary artery bypass "fasttrack" protocol is practical and realistic in a rural environment. Ann Thorac Surg 1997;64:706-9.

10. Velaso FT, Ko W, Rosengart T, Altorki N, Lang S, Gold JP, et al. Cost containment in cardiac surgery: results with a critical pathway for coronary bypass surgery at the New York Hospital-Cornell Medical Center. Best Pract Benchmarking Healthcare 1996;1:21-8.

11. Weintraub WS, Craver JM, Jones EL, Golt JP, Deaton C, Culler $\mathrm{SD}$, et al. Improving cost and outcome of coronary surgery. Circulation 1998;98(Suppl):II-23-8.

12. Loop FD, Higgins TL, Panda R, Pearce G, Estafanous FG. Myocardial protection during cardiac operations: decreased morbidity and lower cost with blood cardioplegia and coronary sinus perfusion. J Thorac Cardiovasc Surg 1992;104:608-18.

13. The Society of Thoracic Surgeons National Cardiac Surgery Database. Manual for data managers. Minneapolis: Summit Medical Systems; 1995.

14. Aldea GS, Doursounian M, O'Gara P, Treanor P, Shapira CM, Lazar HL, et al. Heparin-bonded cardiopulmonary bypass circuits and a reduced anticoagulation in patients undergoing primary CABG: a prospective randomized study. Ann Thorac Surg 1996;62:410-8

15. Cowper PA, Peterson ED, DeLong ER, Jollis JG, Muhlbaier LH, Mark DB. Impact of early discharge after coronary artery bypass graft surgery on rates of hospital readmission and death. J Am Coll Cardiol 1997;30:908-13.

16. Rosen AB, Humphries J, Muhlbaier LH, Kiefe CI, Kresowik T, Peterson ED. Effect of clinical factors on length of stay after coronary artery bypass surgery: results of the cooperative cardiovascular project. Am Heart J 1999;138:69-77.

17. Smith PK, Smith RL, Muhlbaier LH. Risk stratification for adverse economic outcomes in cardiac surgery. Ann Thorac Surg 1997;64:S61-3.

18. Weintraub WS, Jones EL, Craver J, Guyton R, Cohen C. Determinants of prolonged length of hospital stay after coronary bypass surgery. Circulation 1989;80:276-84.

\section{Discussion}

Dr Richard D. Weisel (Toronto, Ontario, Canada). You have performed a very important study, and I wholeheartedly agree that cost shifting rather than cost savings occurred in your patients. At our institution, we have also been able to shorten the hospital LOS to increase the number of operations that we were able to perform with the same funding.

I do not know how this information should be used. My institution is pleased that we have shifted the cost of postoperative care to another institution. The individuals to whom we have referred our patients have been able to receive additional funding for their care. Therefore, the question I have for you is whether your providers are unhappy with the shifting of costs.

In Canada, we have a one-payer system, which is the provincial government. Therefore, we need to develop a sys- tem of postoperative care that reduces total costs rather than our institutional costs. We performed a prospective randomized trial comparing fast track techniques with routine postoperative care, and we found that the fast track reduced the LOS and hospital costs. However, Dr Davey Cheng, the head of cardiac anesthesia, was able to obtain from the government all the costs for the patients after discharge from the hospital. This included physician costs, hospital costs, and prescription costs for both groups in the randomized trial. He found no increase in costs for patients who were discharged early in Ontario. Do you think you would also find that the fast track system reduced overall costs?

I was impressed with the differences in practice of the individual surgeons at your institution. It is clear that the crusading surgeon who had the shortest LOS discharged his patients to alternate facilities, which perhaps increased the overall cost of their postoperative recovery. The surgeon who kept his patients an additional day in the hospital seemed to have the lowest overall cost. The surgeons who had excessively longer hospital stays did not have the most cost-efficient outcomes.

In Canada, patients tend to stay longer in hospital because they believe it is their right. I believe that a small surcharge for staying in the hospital beyond what is medically necessary would result in a dramatic reduction in our costs. We hope that we can accomplish what was the practice of one of your surgeons. Having the patients spend 1 additional day may prevent them from being transferred to an additional care facility and allow them to be sent home without the need to be readmitted. Then we can reduce costs to a minimum and provide the most efficient recovery from cardiac surgery.

Dr Lazar. I think the answer comes from our presidential address, in that over the past 10 years we actually have been innovators. One of the innovative things that we have done is to have risen to the call of getting patients out of the hospital earlier. However, in doing that, we have actually increased the cost, and this information is known by our third-party payers. If we as surgeons do something about it now, it will be more palatable than if a solution is forced on us. What can we do?

The answer lies in Table VI, which demonstrates some of the reasons why patients were discharged to extended care facilities. One of the major reasons was medical, including pulmonary problems and other issues. When we looked at their LOS in rehabilitation centers, it became clear that many of these patients were staying in rehabilitation centers much longer than they should have.

One solution is to apply all this information we have about fast tracking and targeted LOS to patients who go to rehabilitation facilities, because many of these patients could leave much earlier than they do.

About $25 \%$ of our patients went to rehabilitation facilities because of social reasons. Many of our patients were older women who had outlived their husbands and had no one at home to care for them. I think we can do a better job of identifying these patients preoperatively and actually getting extended family or making other arrangements so they do not have to stay in a rehabilitation center for 2 weeks because there is no one at home to take care of them. 
Finally, when we target an LOS for 5 days and the patient does not quite meet the goals at day 5 but may be able to meet them at day 7, it might be better to prolong the hospital stay for 2 extra days rather than to discharge the patient on day 5 and have him or her spend an extended time at a rehabilitation center. There is a lot of fat in the system that can be trimmed, and the more cost effective we make the total bundling of CABG surgery, the better off we are going to be.

Dr Lawrence H. Cohn (Boston, Mass). The implication of your paper is that the cost is greater if other health care facilities are used, but you never actually told us about the cost. Is the actual cost of going to a secondary health care facility, even if you have a very low incidence of readmissions to the acute hospital, less if you have a short acute hospital stay and use secondary health facilities such as a skilled nursing center? In other words, did you look at the actual dollar cost of these different ways of handling patients after CABG?

Dr Lazar. The reason we concluded that the apparent cost savings are less is that it is not possible to come up with actual dollars and cents. When we actually looked to see how much it costs to stay at a skilled nursing facility or a rehabilitation center, we found that everyone has a different contract with different coverage. Some are covered for 14 days, some less, and some are paid per diem. We do not actually have the specific amounts.

What we can say is that the reputed cost savings from sending patients home early, or at least discharging them early from the hospital, may not all be realized because some of this money is expended in an extended care facility. If we can reduce those costs, or maybe keep a patient in the hospital a day or 2 longer and then send him or her home, I think we actually will be saving money.

Dr Richard M. Engelman (Springfield, Mass). I congratulate the author on presenting a subject that is important but that we tend to avoid in most circumstances. It clearly is deserving of discussion. In 1994 I published the fast track approach for the patient undergoing CABG based on our experience at Bay State Medical Center in Springfield, Massachusetts, and our coauthor's experience at Hartford Hospital. Having reviewed Dr Lazar's manuscript, I can accurately compare our own experience from a 1992 patient population published in 1994 and his 1998 patient group. More than $90 \%$ of our patients in 1992 were discharged to home and not to an extended care facility. I question what has happened to our present patient population to make them require rehabilitation so frequently and admission to a skilled nursing facility, a phenomena that is not unique to Boston. My initial premise was that the patients we see now are much sicker than they were in 1992.
I therefore decided, on the basis of this manuscript, to compare the 2 patient groups. First, our patients in 1992 were essentially the same age, 64.7 compared with 64.8 . Left ventricular ejection fraction was $47 \%$ for our patients and $49 \%$ for his, obviously not much difference there. I thought maybe the patients had had a higher incidence of infarction in more recent years: indeed, in Dr Lazar's series it was 53\% and in 1992 it was $52 \%$. Clearly, this is not that significant a difference.

Were intra-aortic balloon pumps used more often in 1998 ? The answer is no, $7 \%$ versus $6 \%$. Were we doing more emergency or urgent operations in 1992 than Dr Lazar was in 1998? The 1998 figure was 58\% and the 1992 figure, 53\%. Clearly, this is not a significant difference.

I would ask Dr Lazar for an explanation for this change in behavior, which I, like he, cannot see as being cost effective. Someone must be paying for the services, whether Medicare or some other third party, and this was simply either not available or not apparent to care givers in 1992.

Second, the patients might not desire to remain at home as against being in some kind of a skilled nursing facility if given the choice. Perhaps some mechanism should be developed so that they could be cared for with some additional help at home rather than have to be transferred into a skilled nursing facility. Dr Lazar, what do you think about the outpatient forms of postoperative care vis-a-vis admission to a skilled nursing facility?

Dr Lazar. Thank you, Dr Engelman. I think what you are asking is why are we sending so many more patients to extended care facilities. I agree that, despite some changes, the patient populations are not particularly different. I think the answer is that your pioneering work with fast tracking, which was initially applied to good-risk patients, has been extended to all patients, with the realization that the hospitals can save money by discharging patients earlier. This has prompted many surgical groups around the country to do just that.

There has also been an increase in the number of rehabilitation centers. Rehabilitation centers used to be truly places where patients were sent for rehabilitation. Now we have skilled nursing facilities and transitional care units, which actually act like step-down units except that they are not in your own hospital and your own hospital does not have to pay the health care expenses.

I agree that we can make more use of extended home facilities with Visiting Nurse Association services. Keeping a patient in the hospital a day or 2 longer may annoy the hospital administrators, but I think it will save a lot of money and make it easier for us to negotiate with third-party payers, who are already aware of these cost shifting practices. 